\title{
Decreased VMAT2 in the pancreas of humans with type 2 diabetes mellitus measured in vivo by PET imaging
}

\author{
Gary W. Cline ${ }^{1}$ (I) $\cdot$ Mika Naganawa ${ }^{1} \cdot$ Laigao Chen $^{2} \cdot$ Kristin Chidsey $^{2} \cdot$ Santos Carvajal-Gonzalez $^{2} \cdot$ Sylvester Pawlak $^{2}$. \\ Michelle Rossulek ${ }^{2} \cdot$ Yanwei Zhang $^{2}$ - Jason Bini ${ }^{1} \cdot$ Timothy J. McCarthy $^{2} \cdot$ Richard E. Carson $^{1} \cdot$ Roberto A. Calle ${ }^{2}$
}

Received: 12 December 2017 / Accepted: 29 March 2018 / Published online: 2 May 2018

(C) Springer-Verlag GmbH Germany, part of Springer Nature 2018

\begin{abstract}
Aims/hypothesis The progressive loss of beta cell function is part of the natural history of type 2 diabetes. Autopsy studies suggest that this is, in part, due to loss of beta cell mass (BCM), but this has not been confirmed in vivo. Non-invasive methods to quantify BCM may contribute to a better understanding of type 2 diabetes pathophysiology and the development of therapeutic strategies. In humans, the localisation of vesicular monoamine transporter type 2 (VMAT2) in beta cells and pancreatic polypeptide cells, with minimal expression in other exocrine or endocrine pancreatic cells, has led to its development as a measure of BCM. We used the VMAT2 tracer $\left[{ }^{18} \mathrm{~F}\right]$ fluoropropyl-(+)-dihydrotetrabenazine to quantify BCM in humans with impaired glucose tolerance (prediabetes) or type 2 diabetes, and in healthy obese volunteers (HOV).

Methods Dynamic positron emission tomography (PET) data were obtained for $4 \mathrm{~h}$ with metabolite-corrected arterial blood measurement in $16 \mathrm{HOV}$, five prediabetic and 17 type 2 diabetic participants. Eleven participants (six HOV and five with type 2 diabetes) underwent two abdominal PET/computed tomography (CT) scans for the assessment of test-retest variability. Standardised uptake value ratio (SUVR) was calculated in pancreatic subregions (head, body and tail), with the spleen as a reference region to determine non-specific tracer uptake at 3-4 h. The outcome measure SUVR minus 1 (SUVR-1) accounts for non-specific tracer uptake. Functional beta cell capacity was assessed by C-peptide release following standard (arginine stimulus test [AST]) and acute insulin response to the glucose-enhanced AST (AIRargMAX). Pearson correlation analysis was performed between the binding variables and the C-peptide AUC post-AST and post-AIRargMAX.

Results Absolute test-retest variability (aTRV) was $\leq 15 \%$ for all regions. Variability and overlap of SUVR-1 was measured in all groups; HOV and participants with prediabetes and with type 2 diabetes. SUVR-1 showed significant positive correlations with AIRargMAX (all groups) in all pancreas subregions (whole pancreas $p=0.009$ and pancreas head $p=0.009$; body $p=0.019$ and tail $p=0.023)$. SUVR-1 inversely correlated with $\mathrm{HbA}_{1 \mathrm{c}}$ (all groups) in the whole pancreas $(p=0.033)$ and pancreas head $(p=$ $0.008)$. SUVR-1 also inversely correlated with years since diagnosis of type 2 diabetes in the pancreas head $(p=0.049)$ and pancreas tail $(p=0.035)$.

Conclusions/interpretation The observed correlations of VMAT2 density in the pancreas and pancreas regions with years since diagnosis of type 2 diabetes, glycaemic control and beta cell function suggest that loss of BCM contributes to deficient insulin secretion in humans with type 2 diabetes.
\end{abstract}

Keywords Beta cell mass $\cdot$ Pancreas $\cdot$ PET imaging $\cdot$ Type 2 diabetes $\cdot$ VMAT2

Electronic supplementary material The online version of this article (https://doi.org/10.1007/s00125-018-4624-0) contains peer-reviewed but unedited supplementary material, which is available to authorised users.

Gary W. Cline

gary.cline@yale.edu

1 Yale University, 801 Howard Avenue, PO Box 208048, New Haven, CT 06520, USA

2 Pfizer Worldwide R\&D, Cambridge, MA, USA

Abbreviations
AST
AIRarg
AIRargMAX
aTRV
BCM
COV

Arginine stimulus test

Acute insulin response to arginine for the standard AST

Acute insulin response to the glucose-enhanced AST

Absolute test-retest variability

Beta cell mass

Coefficient of variation 


\section{Research in context}

\section{What is already known about this subject?}

- The number of pancreatic insulin-positive beta cells is variable in humans

- A loss of beta cell mass occurs in some humans with type 2 diabetes

- Positron emission tomography (PET) imaging of vesicular monoamine transporter type 2 (VMAT2), which co-localises with insulin-positive beta cells, may serve as a measure of beta cell mass

\section{What is the key question?}

- Does loss of beta cell mass contribute to deficient glucose-stimulated insulin secretion in humans with type 2 diabetes?

\section{What are the new findings?}

- Pancreatic VMAT2 correlates with beta cell function across age-BMI-matched healthy obese, insulin-resistant and type 2 diabetic participants

- Pancreatic VMAT2 negatively correlates with glycaemic control and years since diagnosis of diabetes

How might this impact on clinical practice in the foreseeable future?

- Preservation of beta cell mass in people with type 2 diabetes may improve long-term glycaemic control

$\begin{array}{ll}\text { CT } & \text { Computed tomography } \\ \text { FBG } & \begin{array}{l}\text { Fasting blood glucose } \\ { }^{18} \text { F-FP-(+)-DTBZ }\end{array} \\ \begin{array}{l}{\left[{ }^{18} \text { F }\right] \text { fluoropropyl-(+)- }} \\ \text { dihydrotetrabenazine }\end{array} \\ \text { HOV } & \text { Healthy obese volunteer } \\ \text { MRI } & \text { Magnetic resonance imaging } \\ \text { PCRU } & \text { Pfizer Clinical Research Unit } \\ \text { PET } & \text { Positron emission tomography } \\ \text { PP-(cells) } & \text { Pancreatic polypeptide (cells) } \\ \text { ROI } & \text { Region of interest } \\ \text { SUV } & \text { Standardised uptake value } \\ \text { SUVR } & \text { Standardised uptake value ratio } \\ \text { TRV } & \text { Test-retest variability } \\ \text { VMAT2 } & \text { Vesicular monoamine transporter type 2 }\end{array}$

\section{Introduction}

Hyperglycaemia in type 2 diabetes mellitus requires beta cell failure, aggravated by a preceding period of hepatic and peripheral insulin resistance. With the development of impaired glucose tolerance (prediabetes), glycaemic control is maintained by a compensatory increase in insulin secretion, attributed in part to an increase in beta cell mass (BCM). In agreement with rodent studies [1], BCM measured from cadaverous pancreas was greater in obese insulin-resistant humans compared with non-insulin-resistant individuals, but was reduced by $\sim 56 \%$ in comparison with age-BMI-matched donors with type 2 diabetes [2]. However, analysis of islet beta cell composition by electron microscopy found that analysis of an insulin-positive area by immunohistochemistry may overestimate beta cell loss [3]. Analysis using large-scale image capture to obtain a comprehensive view of regional islet size, distribution and cellular composition in cadaveric whole pancreatic sections from donors with type 2 diabetes and nondiabetic donors of similar age and BMI to the obese groups of the earlier study measured an overall reduction of only 15$20 \%$ in beta cells [4]. Thus, the correspondence between loss of BCM and deficiencies in insulin secretion and the timeline of changes in BCM in relation to the development of type 2 diabetes are still open questions.

The recent development of non-invasive in vivo imaging of pancreatic BCM presents the possibility of directly measuring beta cell compensation and loss [5-10]. Molecular imaging of targets that are sufficiently enriched in beta cells compared with other islet and exocrine cell types offers a viable strategy to measure pancreatic BCM $[11,12]$. Our recent work has focused on positron emission tomography (PET) imaging of vesicular monoamine transporter type 2 (VMAT2) as a measure of BCM. Importantly, its use as a biomarker of BCM is supported by the finding that co-expression of VMAT2 and insulin in beta cells was not different in individuals with type 1 or type 2 diabetes compared with those without diabetes [13]. Clinical studies targeting VMAT2 with the PET radioligand $\left[{ }^{18} \mathrm{~F}\right]$ fluoropropyl-(+)-dihydrotetrabenazine $\left({ }^{18} \mathrm{~F}-\mathrm{FP}-(+)-\right.$ DTBZ or $\left.{ }^{18} \mathrm{~F}-\mathrm{AV}-133\right)$ measured decreases in ${ }^{18} \mathrm{~F}-\mathrm{FP}-(+)-$ DTBZ pancreatic uptake (38\% [6]) and binding (40\% [6] and $26 \%$ [10]) in humans with long-standing type 1 diabetes. Taking into account the decrease in pancreas volume in the participants with type 1 diabetes, total pancreatic binding was 
reduced by $59 \%$ [6] and 63\% [10] in the participants with type 1 diabetes. However, as the participants with type 1 diabetes had no measurable beta cell function, the presence of residual background signal raises concerns regarding the beta cell specificity of ${ }^{18} \mathrm{~F}-\mathrm{FP}-(+)$-DTBZ and its utility in quantifying changes in BCM $[14,15]$. However, the contribution of specific and non-specific binding of ${ }^{18} \mathrm{~F}-(+)-\mathrm{FP}-\mathrm{DTBZ}$ to exocrine and endocrine pancreas of animals and humans [16-18], and the localisation of VMAT2 to insulin-positive beta cells and other cell populations (e.g. pancreatic polypeptide [PP]-cells) [11, 13], have been evaluated and indicate that changes in pancreatic binding of ${ }^{18} \mathrm{~F}-\mathrm{FP}-(+)-\mathrm{DTBZ}$ track changes in BCM. Interestingly, residual background was also present in the pancreas of most of the individuals with type 1 diabetes participating in imaging studies that used radioligands targeting alternative indicators of $\mathrm{BCM}$ (serotonin biosynthesis [9] and glucagon-like peptide 1 receptor [GLP-1R] [8]). The common observation of residual background signal using three different classes of imaging agents in individuals with type 1 diabetes supports the possibility that these agents are revealing novel aspects of beta cell biology within the context of health and diabetes [14].

Recent imaging studies to assess changes in BCM in humans with type 2 diabetes indicate that beta cell loss is less than earlier immunohistochemical analyses reported. Studies using ${ }^{18} \mathrm{~F}-\mathrm{FP}-(+)$-DTBZ PET measured a 19\% ( $p=\mathrm{NS}, n=3$ ) reduction in pancreatic binding [10], while pancreatic binding of $\left[{ }^{11} \mathrm{C}\right] 5$-hydroxy-tryptophan in participants with type 2 diabetes was variable, overlapped with the healthy control participants and did not correlate with changes in beta cell function [7]. To address the divergent results and provide additional insight into changes in BCM that occur with the development of type 2 diabetes, PET imaging was used to: (1) determine if PET measures of ${ }^{18} \mathrm{~F}$-FP-(+)-DTBZ in the pancreas differ between individuals with type 2 diabetes and age-BMI-matched healthy obese volunteers (HOV); (2) determine the extent to which ${ }^{18} \mathrm{~F}$-FP-(+)-DTBZ binding variables correlate with beta cell function using the standard and glucose-enhanced arginine stimulus test (AST) [19, 20]; and (3) assess the intrasubject variability (test-retest) of ${ }^{18} \mathrm{~F}-\mathrm{FP}-(+)$-DTBZ binding.

\section{Methods}

\section{Participants}

A total of 38 individuals participated: $16 \mathrm{HOV}$ (14 men and two women), five individuals with prediabetes (three men and two women) and 17 individuals with type 2 diabetes (11 men and six women). HOV had: no history of type 1 or type 2 diabetes or diagnosed prediabetes; $\mathrm{HbA}_{1 \mathrm{c}}<39 \mathrm{mmol} / \mathrm{mol}$ (5.7\%); fasting blood glucose (FBG) $<5.6 \mathrm{mmol} / \mathrm{l}$; and $75 \mathrm{~g}$ OGTT with $2 \mathrm{~h}$ post challenge glucose $<7.8 \mathrm{mmol} / \mathrm{l}$.
Prediabetic participants had no history of type 1 or type 2 diabetes, $\mathrm{HbA}_{1 \mathrm{c}}<48 \mathrm{mmol} / \mathrm{mol}(6.5 \%)$ and evidence of prediabetes from: $\mathrm{FBG} \geq 5.6 \mathrm{but} \leq 7.0 \mathrm{mmol} / \mathrm{l}$ and $2 \mathrm{~h}$ post-OGTT challenge glucose $<7.8 \mathrm{mmol} / \mathrm{l} ; \mathrm{FBG}<5.6 \mathrm{mmol} / \mathrm{l}$ and OGTT with $2 \mathrm{~h}$ glucose $\geq 7.8$ but $\leq 11.1 \mathrm{mmol} / \mathrm{l}$; or $\mathrm{FBG} \geq 5.6$ but $\leq 7.0 \mathrm{mmol} / 1$ with an OGTT with $2 \mathrm{~h}$ glucose $\geq 7.8$ but $\leq 11.1 \mathrm{mmol} / 1$. Participants with type 2 diabetes had a diagnosis of type 2 diabetes as defined by the ADA criteria, $\mathrm{HbA}_{1 \mathrm{c}}$ $\geq 53 \mathrm{mmol} / \mathrm{mol}(7.0 \%)$ and were treated with lifestyle and one or more glucose-lowering agents. The average age, BMI, time since diagnosis of type 2 diabetes and $\mathrm{HbA}_{1 \mathrm{c}}$ are summarised in Table 1 . The study was approved by the Yale University Human Investigation Committee and the Yale-New Haven Hospital Radiation Safety Committee and in accordance with federal guidelines and regulations of the USA for the protection of human research subjects contained in Title 45 Part 46 of the Code of Federal Regulations (45 CFR 46). All participants signed a written informed consent.

\section{Study design}

Each participant underwent one abdominal magnetic resonance imaging (MRI) scan and at least one abdominal PET/ computed tomography (CT) scan. Of 38 participants, 11 participants (six HOV and five with type 2 diabetes) underwent two abdominal PET/CT scans for the assessment of test-retest variability (TRV). Test-retest scans were acquired 7-15 days apart. The remaining participants had only one PET/CT scan. Each participant had up to five visits: screening, PET/CT scan(s), MRI scan and AST. All participants underwent both standard and glucose-enhanced AST to examine insulin secretory capacity. The PET imaging took place at the Yale University PET Center $3 \pm 0.2$ days after the AST, and the MRI imaging took place at the Yale Magnetic Resonance Research Center $5 \pm 0.2$ days after the AST. All screening activities and AST procedures were performed at the New Haven Pfizer Clinical Research Unit (PCRU).

\section{Arginine stimulus test}

The beta cell function of all participants was determined by the acute C-peptide response to arginine stimulation and glucose-enhanced arginine stimulation [19, 20]. Participants were admitted to the PCRU and fasted overnight for at least $8 \mathrm{~h}$ prior to AST. Participants with type 2 diabetes had their oral glucose-lowering agents and/or insulin dose withheld on the morning of the AST procedure. Two antecubital venous catheters were inserted, one for arginine and glucose injection and the other for collection of fasting blood samples to determine the levels of glucose, C-peptide and other hormones. After collection of baseline samples $(-10,-5$ and 0 min prior to arginine bolus), the standard AST (executed at the participant's basal glucose level) was initiated with an intravenous 
Table 1 Participant demographics and beta cell function

\begin{tabular}{|c|c|c|c|c|c|c|c|}
\hline Diagnosis & Age (years) & BMI $\left(\mathrm{kg} / \mathrm{m}^{2}\right)$ & $\begin{array}{l}\text { Time since T2DM } \\
\text { diagnosis (years) }\end{array}$ & $\begin{array}{l}\mathrm{HbA}_{1 \mathrm{c}} \\
(\mathrm{mmol} / \mathrm{mol})\end{array}$ & $\mathrm{HbA}_{1 \mathrm{c}}(\%)$ & $\begin{array}{l}\text { AIRarg } \\
(\mathrm{ng} / \mathrm{ml})\end{array}$ & $\begin{array}{l}\text { AIRargMax } \\
(\mathrm{ng} / \mathrm{ml})\end{array}$ \\
\hline $\mathrm{HOV}(n=16)$ & $51 \pm 4$ & $29 \pm 2$ & - & $35 \pm 2 * * *$ & $5.3 \pm 0.2 * *$ & $2.4 \pm 1.1$ & $9.9 \pm 3.1 * * *$ \\
\hline Prediabetes $(n=5)$ & $56 \pm 5$ & $30 \pm 3$ & - & $33 \pm 4 * * *$ & $5.2 \pm 0.4 * *$ & $3.3 \pm 0.7 * *$ & $9.6 \pm 2.5 * * *$ \\
\hline $\mathrm{T} 2 \mathrm{DM}(n=17)$ & $55 \pm 6$ & $30 \pm 2$ & $9.6 \pm 5.8$ & $69 \pm 19$ & $8.5 \pm 1.7$ & $1.8 \pm 1.3$ & $3.9 \pm 2.8$ \\
\hline
\end{tabular}

Data are mean $\pm \mathrm{SD}$

$* * p<0.01$ vs T2DM, ***p $<0.001$ vs T2DM

T2DM, type 2 diabetes

bolus injection of $5 \mathrm{~g}$ arginine hydrochloride $10 \%$ (wt/vol.) administered over a period of $\sim 1 \mathrm{~min}$, with blood samples collected for glucose, insulin and C-peptide over the next $10 \mathrm{~min}(2,3,4,5,6$ and $10 \mathrm{~min}$ after arginine administration). Immediately following the collection of the $10 \mathrm{~min}$ sample, glucose levels were elevated by a continuous infusion of glucose (as dextrose $20 \%$ in water) at a rate of $900 \mathrm{mg} / \mathrm{min}$ for 70 min. After collecting new baseline samples at 50, 55 and $60 \mathrm{~min}$ after the start of the glucose infusion, the glucoseenhanced AST was initiated with an intravenous bolus injection of $5 \mathrm{~g}$ arginine hydrochloride $10 \%$ (wt/vol.). Timed blood samples were again collected for glucose, insulin and Cpeptide over the next $10 \mathrm{~min}$ at the same intervals.

The acute insulin response to arginine for the standard AST (AIRarg) was defined as the mean of the three highest Cpeptide values from $2,3,4$ and 5 min minus baseline $\mathrm{C}$ peptide (average of $-10,-5$ and $0 \mathrm{~min}$ ). The acute insulin response to the glucose-enhanced AST (AIRargMAX) was defined as the mean of the three highest $\mathrm{C}$-peptide values at 62, 63, 64 and 65 min minus baseline C-peptide at elevated glucose (average of 50, 55 and $60 \mathrm{~min}$ ) [19, 20].

\section{Pancreas volume by MRI}

Pancreas volume was determined as previously described [6]. A magnetic resonance image of the trunk was acquired using a Siemens Sonata 1.5 T Instrument (multi-breath-hold T1weighted acquisition, field of view $38.0 \times 38.0 \mathrm{~cm}$, matrix $256 \times 256$, in-plane resolution $1.48 \mathrm{~mm}, 50$ contiguous slices, $5 \mathrm{~mm}$ slice thickness). Interactive level detection was used with a threshold to distinguish pancreas from surrounding tissue and pancreas regions of interest (ROIs) were delineated on axial sections and confirmed in orthogonal planes using the BioImage Suite software, version 3.01 (https://medicine.yale. edu/bioimaging/suite/). Volumes in each section were summed for total pancreas volume.

\section{Radiosynthesis}

${ }^{18}$ F-FP-(+)-DTBZ was synthesised by Avid Radiopharmaceuticals (Philadelphia, PA, USA) using a synthetic scheme similar to that previously described [17]. The specific activity at time of injection and injected mass were $58 \pm 46 \mathrm{MBq} /$ nmol and $3.1 \pm 1.9 \mu \mathrm{g}$, respectively.

\section{Administration of ${ }^{18} \mathrm{~F}-\mathrm{FP}-(+)-\mathrm{DTBZ}$}

Each participant was limited to a maximum dose of $296 \mathrm{MBq}$ per scan. The mass dose did not exceed $10 \mu \mathrm{g}$ per administration. ${ }^{18}$ F-FP-(+)-DTBZ was administered intravenously over 1 min by infusion pump.

\section{PET imaging and variables}

The mean injected dose of ${ }^{18} \mathrm{~F}-\mathrm{FP}-(+)-\mathrm{DTBZ}$ for all studies was $256 \pm 46 \mathrm{MBq}$. The injected doses at the test and retest scans were $259 \pm 25$ and $270 \pm 28 \mathrm{MBq}$, respectively. Administered doses, specific activities and injected mass were not statistically significantly different between test and retest scans (two-sided paired $t$ test, $p=0.10, p=0.36$ and $p=0.74$, respectively). PET images were acquired using a Biograph mCT PET/CT scanner (Siemens Medical Systems, Knoxville, TN, USA). Dynamic scan data were reconstructed with the ordered subset expectation maximisation algorithm with point spread function correction using time-of-flight measurements.

The PET study consists of a $2 \mathrm{~h}$ scan (SCAN-A) and a $1.5 \mathrm{~h}$ scan (SCAN-B), with a 30 min break between the two scans. The frame timing of PET data was $6 \times 30 \mathrm{~s}, 3 \times 1 \mathrm{~min}, 2 \times$ $2 \mathrm{~min}$ and $22 \times 5 \mathrm{~min}(\mathrm{SCAN}-\mathrm{A})$ and $18 \times 5 \mathrm{~min}(\mathrm{SCAN}-\mathrm{B})$.

PET images were corrected for body motion using a mutualinformation-based algorithm (BioImage Suite software, version 3.01, https://medicine.yale.edu/bioimaging/suite/).

\section{Image analysis}

Computation of regional time-activity curves Regions of interest were manually delineated on the summed image ( 0 $90 \mathrm{~min}$ ) of motion-corrected SCAN-A: pancreas head, pancreas body, pancreas tail, kidney (left and right) and spleen. The summed PET image (150-240 min) of SCAN-B was coregistered to the summed PET image (0-90 min) of SCAN-A using a mutual-information-based algorithm. The drawn ROIs 
were warped to SCAN-B using the estimated transformation, and edited manually in the case of misregistration between SCAN-A and SCAN-B. ROIs were thinned using the 'classical thinning algorithm' [21] to minimise partial volume and respiratory motion effects. Pancreas subregions were concatenated to form the whole pancreas ROI. The final ROIs were applied to the images (electronic supplementary material [ESM] Fig. 1) to generate regional time-activity curves (ESM Fig. 2).

The spleen was clearly seen in the PET images. As the upper (superior) part of the spleen is more susceptible to errors from body motion (e.g. because of attenuation mismatch), the spleen ROI was located on the lower (inferior) part of the spleen. For kidney ROI placement, as the lower part of the kidney can be out of the field of view and the upper part of the kidney can be influenced by spill-in from the uptakes in neighbouring organs, the kidney ROI was located on the middle part of the kidney.

PET quantification Regional standardised uptake value ratio (SUVR) was computed from the time window (180-240 min) as the ratio of the standardised uptake value (SUV) from two different regions (from a target and a reference region). When uptake in the target region is equal to that in the reference region, the value of SUVR is 1 . The outcome measure in this study is SUVR minus 1 (SUVR-1), where the subtraction of 1 accounts for non-specific tracer uptake. In previous human or non-human primate studies $[6,18]$ the spleen and kidney have been proposed as candidate reference regions. However, we recently determined that the minor contribution of radiometabolites relative to the parent ${ }^{18} \mathrm{~F}-\mathrm{FP}-(+)$-DTBZ, and dimensions that minimise partial volume effects, makes the spleen a more practical pseudo-reference region [17].

Outcome measures were compared between HOV and the participants with prediabetes or type 2 diabetes using twotailed unpaired $t$ test.

The mean and SD of TRV were calculated using the following formula:

$\mathrm{TRV}=100 \times \frac{\text { retest }- \text { test }}{(\text { test }+ \text { retest }) / 2}$

The absolute value of TRV (aTRV) was also calculated. The mean of TRV is an index of trends in the outcome measures between the two scans, and the SD of TRV is an index of the variability of the percentage difference between the two measurements. The aTRV combines these two effects into a single value.

\section{Statistical analysis}

Quantitative results are presented as mean $\pm \mathrm{SD}$. Differences between groups were analysed using a two-tailed $t$ test, assuming equal variance. Relationships between SUVR-1 and beta cell function as measured by the AST, $\mathrm{HbA}_{1 \mathrm{c}}$ and years of diabetes were assessed using the Pearson correlation coefficient. A value of $p<0.05$ was considered statistically significant. All tests except for power analysis were performed using GraphPad Prism 7 software. Power analysis was conducted using G*Power Version 3.1.9.2.

\section{Results}

\section{Arginine stimulus test}

Plasma glucose levels after an overnight fast and immediately prior to the AST were higher in the participants with type 2 diabetes $(7.8 \pm 2.3 \mathrm{mmol} / \mathrm{l})$ compared with the $\mathrm{HOV}(5.1 \pm$ $0.3 \mathrm{mmol} / 1, p<0.0005)$ and participants with prediabetes $(5.3$ $\pm 0.3 \mathrm{mmol} / \mathrm{l}, p=0.03$ ). The elevated fasting glucose in those with type 2 diabetes may account for the lack of significance $(p=0.07)$ in the beta cell function as measured by AIRarg (Table 1). However, beta cell function measured with the glucose-enhanced AST, AIRargMax, was significantly decreased in those with type 2 diabetes compared with HOV and participants with prediabetes (Table 1).

\section{Uptake images}

Figure 1 shows typical examples of SUV images of ${ }^{18} \mathrm{~F}$-FP(+)-DTBZ in an HOV and a participant with type 2 diabetes summed from 180 to 240 min post-injection.

\section{Test-retest variability}

The TRV across participants is shown in Fig. 2 and Table 2. The aTRV was $\leq 15 \%$ for all regions.

\section{Outcome measures}

The mean and \% coefficient of variation (COV) of SUVR-1 across participants are shown in Table 3. The rank order of uptake was pancreas head $>$ pancreas body $>$ pancreas tail in all groups.

Comparison of SUVR-1 (180-240 min) values in the subregions of pancreas is shown in Fig. 3. A non-significant decrease in SUVR-1 for all pancreatic subregions was observed in individuals with type 2 diabetes compared with the HOV for all pancreatic subregions, with group differences in the pancreas head approaching significance $(p=0.058)$. Note that there was no difference in spleen SUVs. In fact, the mean spleen SUV was slightly higher than that of the individuals with type 2 diabetes, so the between-group difference in SUVR-1 might be underestimated. 
a

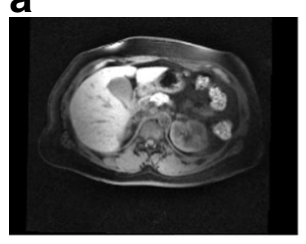

b
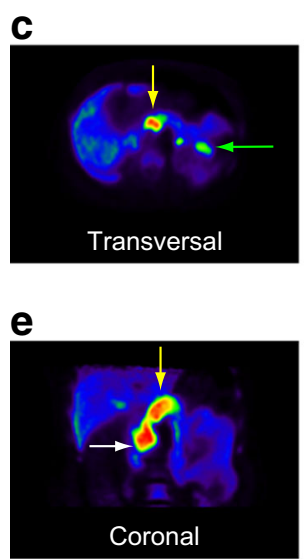

d

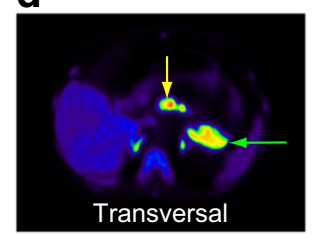

f

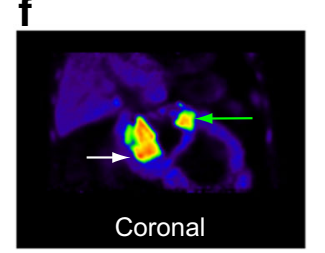

Table 2 SUVR-1 TRV in the pancreas

\begin{tabular}{lllll}
\hline Variability measure & Head & Body & Tail & Whole \\
\hline TRV (\%) & $3 \pm 13$ & $5 \pm 15$ & $3 \pm 18$ & $4 \pm 13$ \\
aTRV (\%) & 12 & 14 & 15 & 12 \\
\hline
\end{tabular}

Data are mean $\pm \mathrm{SD}, n=11$
SUV
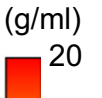

with AIRargMAX (all participants), $\mathrm{HbA}_{1 \mathrm{c}}$ (all participants) and years since diagnosis of type 2 diabetes were observed (Fig. 4). SUVR-1 showed significant positive correlations with AIRargMAX in all pancreas subregions. SUVR-1 was inversely correlated with $\mathrm{HbA}_{1 \mathrm{c}}$ (whole pancreas and pancreas head) and years since diagnosis of type 2 diabetes (pancreas tail and head). Correlation coefficients and $p$ values are summarised in Table 4.

\section{Discussion}

In this study, PET imaging of VMAT-2 with ${ }^{18} \mathrm{~F}-\mathrm{FP}-(+)-$ DTBZ was used to assess the correlation of BCM with function in age-BMI-matched healthy control participants and individuals with type 2 diabetes to test the hypothesis that a loss of BCM contributes to impaired insulin secretion in humans with type 2 diabetes. We observed a large spread of ${ }^{18} \mathrm{~F}-\mathrm{FP}$ (+)-DTBZ binding and uptake variables in the pancreas of the $\mathrm{HOV}$ and prediabetes participants overlapping with the type 2 diabetes participants (Fig. 3, Table 3 and ESM Table 1). However, significant correlations of SUVR-1 were determined for: (1) the whole pancreas and the pancreas head, body and tail with AIRargMax; (2) the whole pancreas and pancreas head with $\mathrm{HbA}_{1 \mathrm{c}}$; and (3) the whole pancreas and pancreas head and tail with years since diagnosis of diabetes (Table 4 , Fig. 4). These results support the hypothesis that a global loss of VMAT2 density (as a measure of BCM) may contribute to deficient insulin secretion in humans with type 2 diabetes, and that a progressive loss of $\mathrm{BCM}$ with duration of type 2 diabetes contributes to poorer glycaemic control. The modest loss of VMAT2 binding in the participants with type 2 diabetes,
Fig. 2 (a) ${ }^{18} \mathrm{~F}-\mathrm{FP}-(+)-\mathrm{DTBZ}$ SUVR-1 (unitless) at test and retest scans. Each symbol denotes each scan. Grey circles, test; white circles, retest. Lines show means \pm SD. (b) Repeated measurement of ${ }^{18}$ F-FP-(+)-DTBZ SUVR-1 in the whole pancreas a

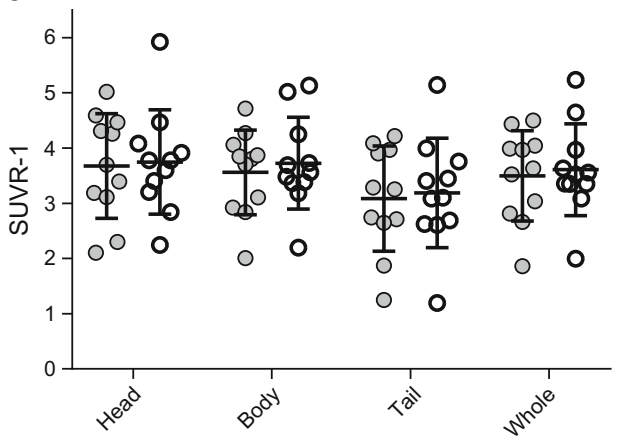

b

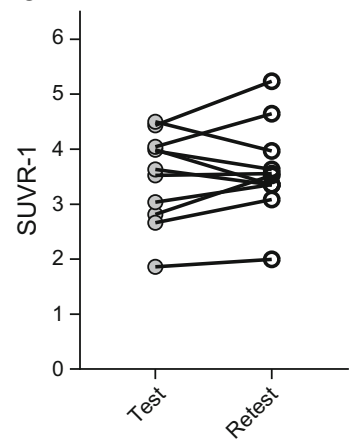


Table 3 Pancreas volume and uptake (SUVR-1) of ${ }^{18} \mathrm{~F}-\mathrm{FP}-(+)-\mathrm{DTBZ}$

\begin{tabular}{llllllll}
\hline Participant & $\begin{array}{l}\text { MRI: pancreas } \\
\text { volume }(\mathrm{ml})\end{array}$ & $\begin{array}{l}\text { Pancreas volume } \\
\text { index }\left(\mathrm{ml} / \mathrm{m}^{2}\right)\end{array}$ & \multicolumn{2}{l}{ Mean SUVR-1 } & & \multicolumn{2}{c}{ SUVR-1 $\times$ volume (ml) } \\
\cline { 5 - 7 } & & & Head & Body & Tail & Whole & Whole \\
\hline HOV $(n=16)$ & $66.4(26)$ & $31.4(25)$ & $4.2(24)$ & $3.8(26)$ & $3.5(30)$ & $3.8(25)$ & $258(43)$ \\
Prediabetes $(n=5)$ & $58.3(30)$ & $28.0(24)$ & $4.3(29)$ & $4.1(31)$ & $3.7(34)$ & $4.0(31)$ & $235(44)$ \\
T2DM $(n=17)$ & $57.8(35)$ & $29.2(33)$ & $3.5^{\text {Il }}(29)$ & $3.5(26)$ & $3.1(28)$ & $3.4(27)$ & $196(47)$ \\
\hline
\end{tabular}

Data are mean $(\% \mathrm{COV})$

II $p=0.058$ vs HOV

T2DM, type 2 diabetes

together with the large overlapping spread in pancreatic VMAT2 binding and beta cell density found here and previously $[4,7,23]$, suggests that loss of beta cell function is primarily responsible for insufficient insulin secretion of humans with type 2 diabetes, with the loss of BCM acting to exacerbate this deficiency.

Co-localisation of VMAT2 with insulin-positive pancreatic islet beta cells in both non-diabetic and individuals with type 2 diabetes [13], combined with the high affinity of ${ }^{18} \mathrm{~F}-\mathrm{FP}-(+)-$ DTBZ for VMAT2 [24, 25], support its use to quantify changes in BCM $[6,18]$. Although the majority of VMAT2 is expressed in beta cells, $40 \%$ of PP cells have been shown to be VMAT2 positive [13], and may contribute to radiotracer binding. In most of the pancreas, PP-cell density ( $\sim 50-100$ times lower than beta cell density) would be expected to make negligible contribution to pancreatic radiotracer uptake [26, 27]. However, the measured volume densities of PP and beta cells in the head region abutting the duodenal wall are similar, and loss of PP-cells, or atrophy in this region with loss of endocrine cell mass, could lead to a measurable reduction in radiotracer binding $[11,27]$. No significant differences in PP-

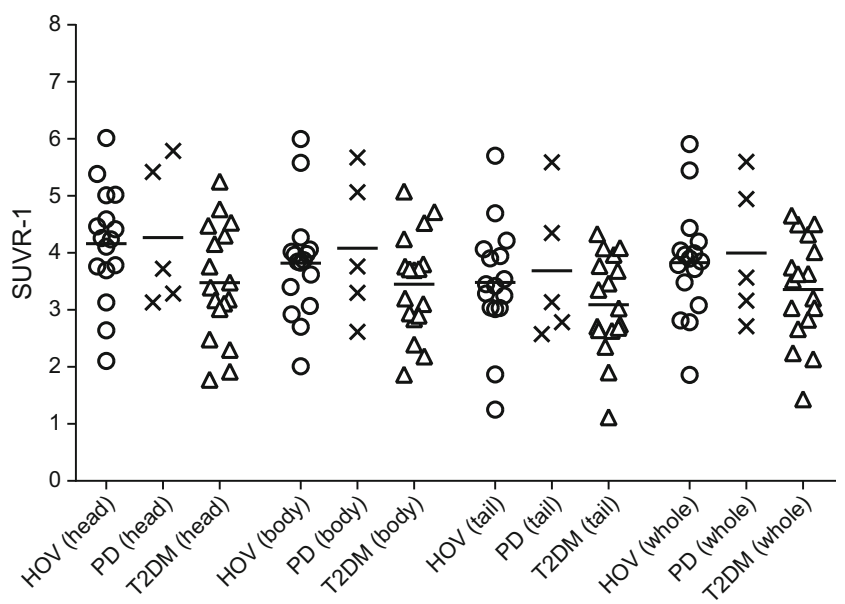

Fig. 3 Comparison of ${ }^{18}$ F-FP-(+)-DTBZ uptake (SUVR-1 [unitless]) in pancreas between $\mathrm{HOV}$, prediabetes and type 2 diabetes groups. Lines show means. Circles, HOV; crosses, prediabetes; triangles, type 2 diabetes. PD, prediabetes; T2DM, type 2 diabetes cell volume density throughout the pancreas, or within the PPcell-rich region of the pancreas head, were observed in control participants and donors with type 2 diabetes (58-75 years) $[22,26]$. Thus, the progressive reduction in ${ }^{18} \mathrm{~F}-\mathrm{FP}-(+)-$ DTBZ binding that we observed in participants with type 2 diabetes is more likely owing to loss of VMAT2-positive beta cells than to loss of PP cells.

We chose the $4 \mathrm{~h}$ scan duration to evaluate the optimum time window for computing SUVR. We found SUVR (180 to $240 \mathrm{~min}$ ) gave the best test-retest reproducibility, and it was therefore chosen as the primary outcome measurement. The TRV for SUVR (180-240 min) was slightly higher than the binding potential (non-displaceable $\left[\mathrm{BP}_{\mathrm{ND}}\right]$ ) for healthy control participants and participants with type 1 diabetes $(9.4 \%)$ [10]. The TRV of SUVR-1 was good $(\leq 15 \%)$ in all pancreatic subregions for both the HOV and those with type 2 diabetes. This value was used to perform a power analysis to detect within-subject change (paired sample, two-tailed $t$ test, statistical power 0.95). Sample sizes of five or 13 participants would be needed to detect within-subject changes of $20 \%$ or $10 \%$, respectively.

There are limitations to our study. Foremost is the assumption that VMAT2 expression is proportional to BCM. As discussed above, VMAT2 expression in PP cells can compromise the correlation of VMAT2 binding with BCM. In addition, the potential for dedifferentiation of beta cells with type 2 diabetes may occur such that changes in the cell's phenotypic expression resulting in decreased functional BCM may occur with or without changes in VMAT2 [28-31]. We used the glucose-enhanced AST to determine maximal beta cell function, rather than measures of beta cell sensitivity to glucose such as graded glucose infusion test [32]. As prior studies have found that obesity, per se, may lead to increased BCM, difference in BCM between obese individuals with type 2 diabetes could be anticipated to be less in comparison with lean than with obese healthy individuals [2]. We selected ageBMI-matched participants to maximise BCM differences. Although the smaller group size for those with prediabetes $(n=5)$, in comparison with the type 2 diabetes $(n=16)$ and HOV ( $n=17)$ groups, limited our ability to detect the 
Fig. $4{ }^{18}$ F-FP-(+)-DTBZ uptake is positively correlated with glucose-potentiated C-peptide release (AIRargMAX) and inversely correlated with glycaemic control $\left(\mathrm{HbA}_{1 \mathrm{c}}\right)$ and years since diagnosis of type 2 diabetes in $\mathrm{HOV}$, prediabetes and type 2 diabetic participants. (a) Comparison of SUVR-1 (unitless) in the whole pancreas with AIRargMAX. (b-d)

Comparison of SUVR-1 (unitless) in the pancreas head with: (b) AIRargMAX; (c) $\mathrm{HbA}_{1 \mathrm{c}}$; and (d) years since diagnosis of type 2 diabetes. Dotted lines, 95\% CI for line of regression. Circles, HOV; crosses, prediabetes; triangles, type 2 diabetes. T2DM, type 2 diabetes a

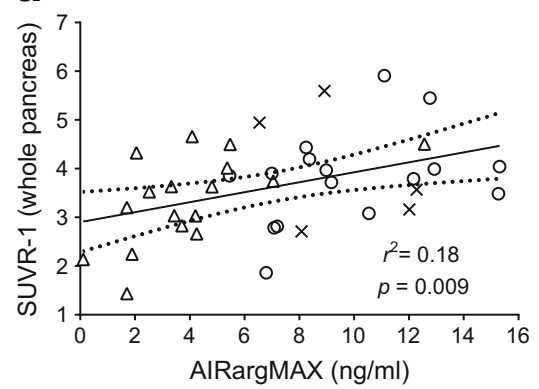

C

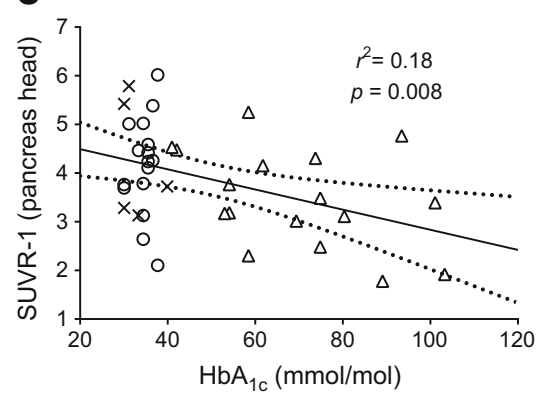

b
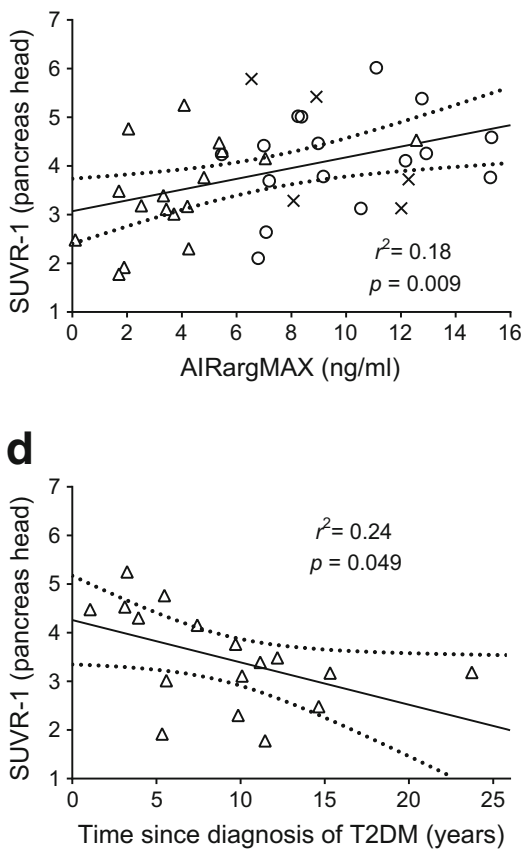

anticipated increase in BCM in prediabetic humans, all three groups were included in assessing the correlation between beta cell function and pancreatic VMAT2 density. Significant overlap, consistent with biological variability of BCM, masked potential group differences in VMAT2 density. Finally, the concern common to all targeted radiotracer imaging studies is the localisation of radiotracer activity to the targeted cell population. Thus, if beta cells represent $\sim 2 \%$ of the pancreas, the localised activity will be $\sim 50$ times higher than the mean pancreas activity.

Studies of cadaverous pancreas obtained from non-diabetic and donors with type 2 diabetes indicate a loss of BCM in those with diabetes, but differ in the magnitude and regional distribution of BCM loss $[2,4,33]$. The heterogeneity in our measured BCM is similar to that observed in a recent PET study using $\left[{ }^{11} \mathrm{C}\right] 5$-hydroxy-tryptophan uptake to measure BCM [7], and with the results from human autopsy studies $[4,23]$. Our results differ from the pronounced decrease in BCM in donors with type 2 diabetes measured by immunohistochemistry in cadaver pancreas [2]. Differences in age, BMI and time since diagnosis of type 2 diabetes may contribute to the disparity between results. The individuals in our study were younger (54 years vs obese 63 years and lean 80 years) and had BMI intermediate between the obese and lean groups in the study of cadaverous pancreas $\left(29 \mathrm{~kg} / \mathrm{m}^{2}\right.$ vs obese $37 \mathrm{~kg} / \mathrm{m}^{2}$ and lean $22 \mathrm{~kg} / \mathrm{m}^{2}$ ) [2]. A similar study measured a $\sim 25 \%$ decrease in beta cell volume density in donors with diabetes [22]. Although our participants were younger (54 years vs 69 years), BMIs were similar (29 kg/ $\mathrm{m}^{2}$ vs $30 \mathrm{~kg} / \mathrm{m}^{2}$ ). In agreement with our results, BCM was found to decrease with time from type 2 diabetes diagnosis, and a large overlapping range of beta cell volume densities ( $\sim 0.25$ to $2.5 \%$ ) was measured for both the non-diabetic and type 2 diabetic donors. In a study of whole pancreatic sections, donors with type 2 diabetes of similar age (58 years vs 55 years), BMI $\left(30 \mathrm{~kg} / \mathrm{m}^{2}\right.$ vs $\left.30 \mathrm{~kg} / \mathrm{m}^{2}\right)$ and time since type 2 diabetes diagnosis (14 years vs 10 years) to our participants had a loss of beta cells localised to the head of the pancreas, with no statistically significant beta cell loss in the pancreas body or tail [4]. Our in vivo PET imaging results are similar, with the greatest mean loss $(\sim 17 \%, p=0.058)$ of VMAT2 in the head of the pancreas of participants with type 2 diabetes.
Table 4 Correlations of SUVR-1 with C-peptide release (AIRargMAX), $\mathrm{HbA}_{1 \mathrm{c}}$ and years since diagnosis of diabetes

\begin{tabular}{|c|c|c|c|c|c|c|}
\hline \multirow[t]{2}{*}{ Region } & \multicolumn{2}{|c|}{ AIRargMAX } & \multicolumn{2}{|l|}{$\mathrm{HbA}_{1 \mathrm{c}}$} & \multicolumn{2}{|c|}{ Years since diagnosis of diabetes } \\
\hline & $r$ & $p$ value & $r$ & $p$ value & $r$ & $p$ value \\
\hline Pancreas head & 0.42 & 0.009 & -0.43 & 0.008 & -0.48 & 0.049 \\
\hline Pancreas body & 0.38 & 0.019 & -0.30 & 0.068 & -0.39 & 0.119 \\
\hline Pancreas tail & 0.37 & 0.023 & -0.28 & 0.086 & -0.51 & 0.035 \\
\hline Whole pancreas & 0.42 & 0.009 & -0.35 & 0.033 & -0.43 & 0.088 \\
\hline
\end{tabular}


In summary, VMAT2 binding correlated: (1) positively with beta cell function for the whole pancreas, and pancreas head, body and tail; (2) inversely with $\mathrm{HbA}_{1 \mathrm{c}}$ for the whole pancreas and pancreas head; and (3) inversely with years since diagnosis with type 2 diabetes for the pancreas head and tail. Thus, progressive reduction of VMAT2 density, indicative of loss of BCM, in the pancreas of individuals with type 2 diabetes may contribute to deficient glucose-stimulated insulin secretion.

Acknowledgements The authors appreciate the excellent technical assistance of staff at the Yale University PET Center, especially PET Technologists D. Ruggiero and E. Hidalgo, and the New Haven PCRU.

Some of the data were presented as an abstract at the ADA 76th Scientific Sessions, 10-14 June 2016, New Orleans, LA, USA.

Data availability All data generated or analysed during this study are included in this published article and its supplementary files.

Funding This work was supported by the Yale-Pfizer Bioimaging Research Alliance and National Institutes of Health (NIH) grant 1S10OD010322-01. This publication was also made possible by Clinical and Translational Science Award Grant Number UL1 TR000142 from the National Center for Advancing Translational Sciences (NCATS), a component of the NIH. Its contents are solely the responsibility of the authors and do not necessarily represent the official view of NIH.

Duality of interest The authors declare that there is no duality of interest associated with this manuscript. The sponsor, Pfizer Worldwide R\&D, was involved in study design and data collection and provided editorial assistance.

Contribution statement All authors contributed to the conception and design of the study/protocol. LC, KC, SC-G, MR and JB researched data and contributed to discussion. GWC, MN and REC wrote the manuscript and researched data. All authors contributed to discussion and reviewed/ edited the manuscript. All authors approved the final version of the manuscript. GWC is the guarantor of this work, had full access to all the data in the study and takes responsibility for the integrity of the data and accuracy of the data analysis.

\section{References}

1. Liu YQ, Jetton TL, Leahy JL (2002) Beta-cell adaptation to insulin resistance. Increased pyruvate carboxylase and malate-pyruvate shuttle activity in islets of nondiabetic Zucker fatty rats. J Biol Chem 277:39163-39168

2. Butler AE, Janson J, Bonner-Weir S, Ritzel R, Rizza RA, Butler PC (2003) Beta-cell deficit and increased beta-cell apoptosis in humans with type 2 diabetes. Diabetes 52:102-110

3. Marselli L, Suleiman M, Masini M et al (2014) Are we overestimating the loss of beta cells in type 2 diabetes? Diabetologia 57:362-365

4. Wang X, Misawa R, Zielinski MC et al (2013) Regional differences in islet distribution in the human pancreas - preferential beta-cell loss in the head region in patients with type 2 diabetes. PLoS One 8: e67454

5. Goland R, Freeby M, Parsey R et al (2009) 11C-dihydrotetrabenazine PET of the pancreas in subjects with long-standing type 1 diabetes and in healthy controls. J Nucl Med 50:382-389
6. Normandin MD, Petersen KF, Ding YS et al (2012) In vivo imaging of endogenous pancreatic beta-cell mass in healthy and type 1 diabetic subjects using $18 \mathrm{~F}$-fluoropropyl-dihydrotetrabenazine and PET. J Nucl Med 53:908-916

7. Carlbom L, Espes D, Lubberink M et al (2017) [11C]5-Hydroxytryptophan PET for assessment of islet mass during progression of type 2 diabetes. Diabetes 66:1286-1292

8. Brom M, Woliner-van der Weg W, Joosten L et al (2014) Noninvasive quantification of the beta cell mass by SPECT with ${ }^{111} \mathrm{In}$ labelled exendin. Diabetologia 57:950-959

9. Eriksson O, Espes D, Selvaraju RK et al (2014) Positron emission tomography ligand [11C]5-hydroxy-tryptophan can be used as a surrogate marker for the human endocrine pancreas. Diabetes 63: 3428-3437

10. Freeby MJ, Kringas P, Goland RS et al (2016) Cross-sectional and test-retest characterization of PET with [(18)F]FP-(+)-DTBZ for beta cell mass estimates in diabetes. Mol Imaging Biol 18:292-301

11. Freeby M, Ichise M, Harris PE (2012) Vesicular monoamine transporter, type 2 (VMAT2) expression as it compares to insulin and pancreatic polypeptide in the head, body and tail of the human pancreas. Islets 4:393-397

12. Harris PE, Ferrara C, Barba P, Polito T, Freeby M, Maffei A (2008) VMAT2 gene expression and function as it applies to imaging betacell mass. J Mol Med 86:5-16

13. Saisho Y, Harris PE, Butler AE et al (2008) Relationship between pancreatic vesicular monoamine transporter 2 (VMAT2) and insulin expression in human pancreas. J Mol Histol 39:543-551

14. Eriksson O, Laughlin M, Brom M et al (2016) In vivo imaging of beta cells with radiotracers: state of the art, prospects and recommendations for development and use. Diabetologia 59:1340-1349

15. Fagerholm V, Mikkola KK, Ishizu T et al (2010) Assessment of islet specificity of dihydrotetrabenazine radiotracer binding in rat pancreas and human pancreas. J Nucl Med 51:1439-1446

16. Singhal T, Ding YS, Weinzimmer D et al (2011) Pancreatic beta cell mass PET imaging and quantification with [11C]DTBZ and [18F]FP-(+)-DTBZ in rodent models of diabetes. Mol Imaging Biol 13:973-984

17. Naganawa M, Lin SF, Lim K et al (2016) Evaluation of pancreatic VMAT2 binding with active and inactive enantiomers of $18 \mathrm{~F}-\mathrm{FP}-$ DTBZ in baboons. Nucl Med Biol 43:743-751

18. Harris PE, Farwell MD, Ichise M (2013) PET quantification of pancreatic VMAT 2 binding using $(+)$ and $(-)$ enantiomers of [(1)(8)F]FP-DTBZ in baboons. Nucl Med Biol 40:60-64

19. Robertson RP, Raymond RH, Lee DS et al (2014) Arginine is preferred to glucagon for stimulation testing of beta-cell function. Am J Phys Endocrinol Metab 307:E720-E727

20. Shankar SS, Vella A, Raymond RH et al (2016) Standardized mixed-meal tolerance and arginine stimulation tests provide reproducible and complementary measures of beta-cell function: results from the Foundation for the National Institutes of Health Biomarkers Consortium Investigative Series. Diabetes Care 39: $1602-1613$

21. Pavlidis T (1982) Algorithms for graphics and image processing. Computer Science Press, Berlin Heidelberg

22. Rahier J, Guiot Y, Goebbels RM, Sempoux C, Henquin JC (2008) Pancreatic beta-cell mass in European subjects with type 2 diabetes. Diabetes Obes Metab 10(Suppl 4):32-42

23. Saisho Y, Butler AE, Manesso E, Elashoff D, Rizza RA, Butler PC (2013) Beta-cell mass and turnover in humans: effects of obesity and aging. Diabetes Care 36:111-117

24. Kilbourn MR, Hockley B, Lee L et al (2007) Pharmacokinetics of [(18)F]fluoroalkyl derivatives of dihydrotetrabenazine in rat and monkey brain. Nucl Med Biol 34:233-237

25. Kung MP, Hou C, Goswami R, Ponde DE, Kilbourn MR, Kung HF (2007) Characterization of optically resolved 9-fluoropropyl- 
dihydrotetrabenazine as a potential PET imaging agent targeting vesicular monoamine transporters. Nucl Med Biol 34:239-246

26. Rahier J, Wallon J, Loozen S, Lefevre A, Gepts W, Haot J (1983) The pancreatic polypeptide cells in the human pancreas: the effects of age and diabetes. J Clin Endocrinol Metab 56:441-444

27. Wang X, Zielinski MC, Misawa R et al (2013) Quantitative analysis of pancreatic polypeptide cell distribution in the human pancreas. PLoS One 8:e55501

28. Spijker HS, Song H, Ellenbroek JH et al (2015) Loss of beta-cell identity occurs in type 2 diabetes and is associated with islet amyloid deposits. Diabetes 64:2928-2938

29. Wang YJ, Schug J, Won KJ et al (2016) Single-cell transcriptomics of the human endocrine pancreas. Diabetes 65:3028-3038
30. Talchai C, Xuan S, Lin HV, Sussel L, Accili D (2012) Pancreatic beta cell dedifferentiation as a mechanism of diabetic beta cell failure. Cell 150:1223-1234

31. Sakano D, Shiraki N, Kikawa K et al (2014) VMAT2 identified as a regulator of late-stage beta-cell differentiation. Nat Chem Biol 10: 141-148

32. Jung SH, Jung CH, Reaven GM, Kim SH (2018) Adapting to insulin resistance in obesity: role of insulin secretion and clearance. Diabetologia 61:681-687

33. Yoon KH, Ko SH, Cho JH et al (2003) Selective beta-cell loss and alpha-cell expansion in patients with type 2 diabetes mellitus in Korea. J Clin Endocrinol Metab 88:2300-2308 